\title{
Thermal and surface core-electron binding-energy shifts in metals
}

\author{
D. M. Riffe, ${ }^{*}$ G. K. Wertheim, D. N. E. Buchanan, and P. H. Citrin \\ AT\& T Bell Laboratories, Murray Hill, New Jersey 07974-0636 \\ (Received 8 July 1991; revised manuscript received 31 October 1991)
}

\begin{abstract}
High-resolution photoemission spectra from the shallow core levels of alkali metals and of In have been obtained between $78 \mathrm{~K}$ and room temperature. The data yield values for the alkali-metal surfaceatom core-level shift and show thermal shifts of comparable size for bulk and surface. The positive surface shifts are due to the spill-out of conduction-electron charge, which is responsible for the surface dipole layer. The surface shifts are in good agreement with values obtained from a Born-Haber cycle expressed in terms of surface energies. The thermal shifts are proportional to the lattice expansion, and arise from both initial-state and final-state effects. As the lattice expands, the Fermi level decreases, decreasing the core-electron binding energy. At the same time, the expansion of the conduction-electron charge increases $r_{s}$, thereby decreasing the potential at the core level and increasing the binding energy. The expansion also decreases the relaxation energy, further increasing the core-electron binding energy. In the alkali metals, the combined potential- and relaxation-energy terms dominate the Fermi-level term, making the shifts positive. In divalent metals the three terms tend to cancel, while in trivalent metals it is the Fermi-level term that dominates, making the shifts negative.
\end{abstract}

\section{INTRODUCTION}

Changes in core-level binding energies (BE's) with temperature have been reported in insulators ${ }^{1}$ and metals. ${ }^{2,3}$ In both types of systems, the BE shifts arise from the thermal expansion of the lattice. In ionic solids, the shifts arise in the initial state and are due to the change of the Madelung potential, which shifts anion and cation levels in opposite directions. In metals, the $\mathrm{BE}$ variations have previously been attributed to a combination of an initial-state shift in the Fermi level and a final-state change in the relaxation energy. ${ }^{2}$ Comparison with band-structure calculations has not yet shown a clear correlation with initial-state effects, yielding results that differ significantly from experiment not only in magnitude but even in sign. ${ }^{3}$

To investigate the mechanisms responsible for the thermal shift in more detail, we have obtained coreelectron photoemission data over a range of temperatures from the alkali metals $\mathrm{Li}, \mathrm{Na}, \mathrm{K}$, and $\mathrm{Rb}$, and the $s p$ metal In. Our interpretation of the measured shifts is based on the formalism used by Hedin and Lundqvist ${ }^{4}$ to calculate core-electron binding energies in the alkali metals. We show that the thermal shift is made up of three contributions, all attributable to thermal expansion. There are two initial-state effects: (i) a change in the Fermi level (chemical potential), which serves as reference level for the measurement of core-electron binding energies, and (ii) a change in the potential due to the valence charge acting on the core electrons. In addition, there is a change in final-state relaxation energy due to the change in the screening length.

The data also provide very reliable values for the surface-atom core-level shifts (SCS's) of the alkali metals. Except for $\mathrm{Li}$, these shifts are in good accord with values obtained from a Born-Haber cycle expressed in terms of surface energies. This approach gives results in much better agreement with experiment than the standard Born-Haber treatment, in which an average $20 \%$ reduction in cohesive energy is assumed for the surface atoms. Finally, we show that the surface-atom core-level shifts can be described in terms of the mechanisms responsible for the thermal shifts, thereby extending our understanding of the SCS's from tight-binding $d$-band to freeelectron-like $s p$ metals.

\section{EXPERIMENTAL DETAILS}

The alkali-metal samples were prepared by condensation of alkali-metal vapor, obtained from commercial SAES Getters sources, onto a $\mathrm{Ni}(100)$ substrate cooled to $78 \mathrm{~K}$. The $\mathrm{Li}$ and $\mathrm{Na}$ samples showed rather diffuse lowenergy-electron-diffraction (LEED) patterns characteristic of multiple-domain (110)-oriented faces. LEED from the $\mathrm{K}$ and $\mathrm{Rb}$ films showed no long-range order. However, the core-level data of these metals are also characteristic of bcc (110)-oriented surfaces in that there is no evidence of a distinguishable subsurface shift. ${ }^{5}$ The lack of observable LEED features for $\mathrm{K}$ (and $\mathrm{Rb}$ ) in this temperature range is attributed to thermal broadening, since LEED from a $\mathrm{K}$ film at $20 \mathrm{~K}$, prepared as in this work, exhibits a well-defined (110) diffraction pattern. ${ }^{6}$

Photoemission data on the alkali metals were obtained on the AT\&T Bell Laboratories-Oregon 6-m toroidalgrating monochromator beamline at the National Synchrotron Light Source. The photoelectron-energy distributions were measured with a $100-\mathrm{mm}$ Vacuum Science Workshop hemispherical analyzer operated with a pass energy of $2 \mathrm{eV}$ for $\mathrm{Na}, \mathrm{K}$, and $\mathrm{Rb}$ and $5 \mathrm{eV}$ for $\mathrm{Li}$, yielding nominal resolutions of 40 and $100 \mathrm{meV}$, respectively. The monochromator resolution was in the range of 50-90 meV, depending on photon energy, giving total instrumental resolutions in the range of $65-75 \mathrm{meV}$ for $\mathrm{Na}$, $\mathrm{K}$, and $\mathrm{Rb}$ and $135 \mathrm{meV}$ for $\mathrm{Li}$. 
An individual run required about two hours, during which time approximately 30 spectra were obtained as the sample warmed up to room temperature after the supply of liquid nitrogen was shut off. Ambient pressure was in the range of $(1-2) \times 10^{-10}$ Torr, dominated by $\mathrm{H}_{2}$. After collection of the $\mathrm{Na}$ and $\mathrm{Rb}$ spectra, the samples were recooled to check for changes due to possible contamination from the background gases. None were observed.

The In sample was prepared by evaporation from a W filament onto a room-temperature $\mathrm{Cu}$ substrate in the measurement chamber of a photoelectron spectrometer equipped with a $\mathrm{He}$ resonance lamp. The In $4 d$ photoemission spectra were taken with $\mathrm{He}$ II resonance radiation at 78 and $295 \mathrm{~K}$.

\section{RESULTS AND ANALYSIS}

The lowest-temperature data for $\mathrm{Li}, \mathrm{Na}, \mathrm{K}$, and $\mathrm{Rb}$ are shown in Fig. 1. All four alkali metals show a positive SCS (shift to higher BE). The bulk and surface contributions are well resolved in the raw data of $L i$ and $R b$, while in the $\mathrm{Na}$ and $\mathrm{K}$ data the surface components are obscured by the overlapping $p_{1 / 2}$ component of the bulk spin-orbit doublet. For $\mathrm{Na}$, the bulk and surface $2 p_{1 / 2}$ components could be removed by spin-orbit stripping. ${ }^{7}$ This procedure was not successful for $K$, however, because the Coster-Kronig decay channel significantly increases the width of the $3 p_{1 / 2}$ component. The In data for 78 and $295 \mathrm{~K}$ are displayed in Fig. 2. The SCS is too small to be resolved. There is no discernible thermal shift; i.e., it is much less than the $12-\mathrm{meV}$ spacing of the data points.

Alternate scans as a function of temperature from data sets for $\mathrm{Li}, \mathrm{Na}, \mathrm{K}$, and $\mathrm{Rb}$ are shown in Figs. 3-6. The $\mathrm{Na}$ data are shown after spin-orbit stripping. The points in the lowest-temperature trace for $\mathrm{Na}$ show that this procedure does not introduce any anomalous structure. For all four alkali metals, a clear shift to higher binding energy is observed as the temperature increases. In every case, the bulk and surface spectra shift by comparable amounts with temperature. The increased phonon broadening at higher temperature is also apparent for each metal. It has been discussed in a previous publication $^{7}$ and will not be considered here.

Quantitative information on the shifts was obtained by fitting the $\mathrm{Li}$ and $\mathrm{Rb}$ scans with two Doniach-Šunjić (DS) lines $^{8}$ and the $\mathrm{Na}$ and $\mathrm{K}$ data with two spin-orbit doublets of DS line shape. A Gaussian width to account for phonon and instrumental broadening was introduced by convolution. The line-shape parameters were independent, except for common Lorentzian lifetime widths for the bulk and surface components and common singularity indices for all lines. In the case of $\mathrm{Na}$, an additional constraint of identical $2 p_{1 / 2}$ and $2 p_{3 / 2}$ lifetime widths was applied. The constraint of identical bulk- and surface-atom lifetime widths is justified by the fact that the Auger transition rates for the core levels measured here are small (lifetime widths are less than $40 \mathrm{meV}$ ), so any differences due to narrower surface densities of states ${ }^{7}$ should be negligible. Indeed, we found that deter- minations of the bulk line positions are very little affected even when the surface lifetime widths were changed by factors as large as 3. The constraint on the singularity index was motivated by theoretical work that showed that bulk and surface indices are almost identical in alkali metals. ${ }^{9}$ The available data in which the bulk and surface lines have comparable intensity do not suffice to test

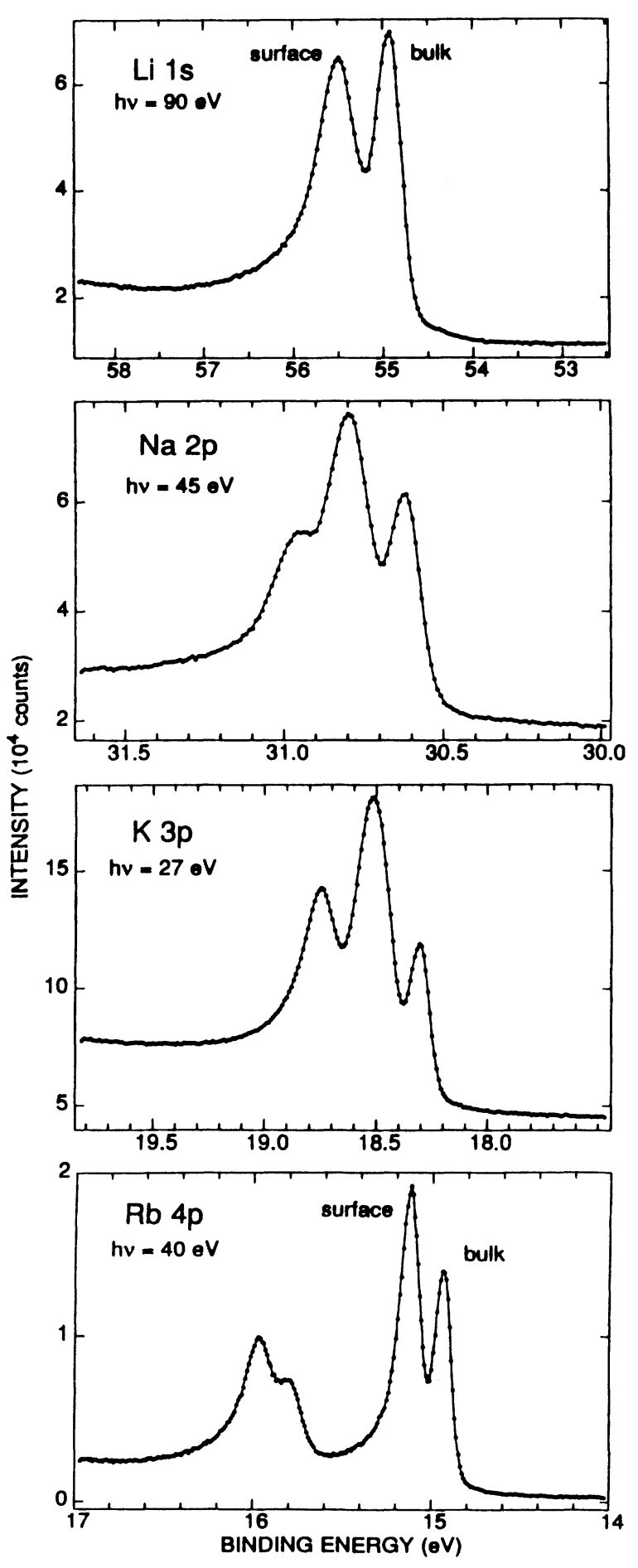

FIG. 1. Photoemission spectra of alkali metals taken with the samples near $80 \mathrm{~K}$. 


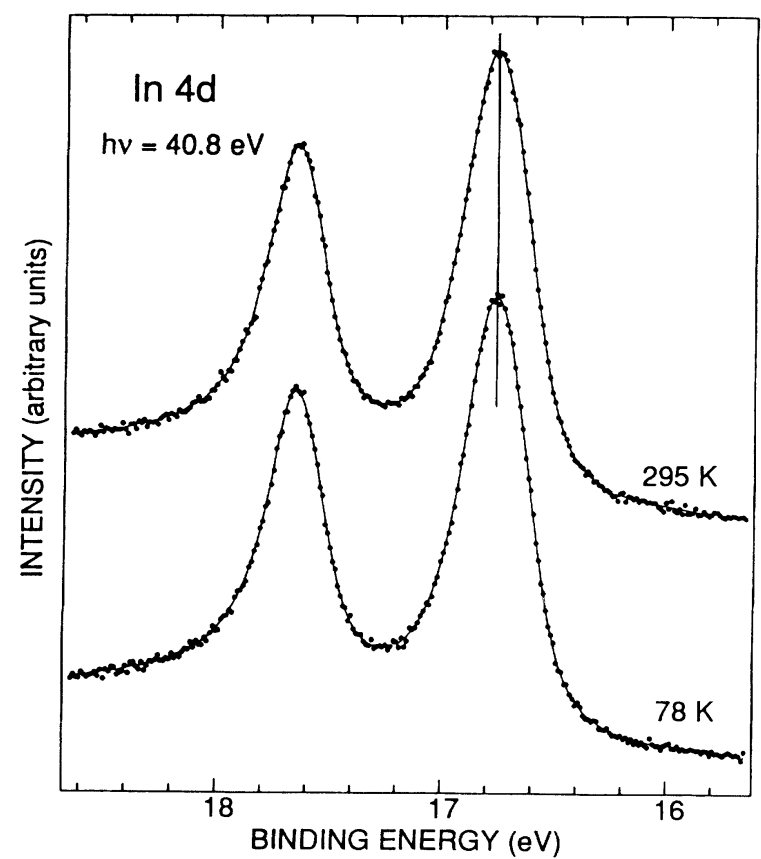

FIG. 2. In $4 d$ photoemission spectra taken with $40.8-\mathrm{eV}$ He II resonance radiation.

this assumption, because the least-squares analysis shows very strong correlations between the two singularity indices when they are given independent values. (Data must be taken over a wide range of escape depths in order to test this assumption critically.) The background, including the low-energy tail of the surface plasmon, was represented by a sloping line and a power-law term. The

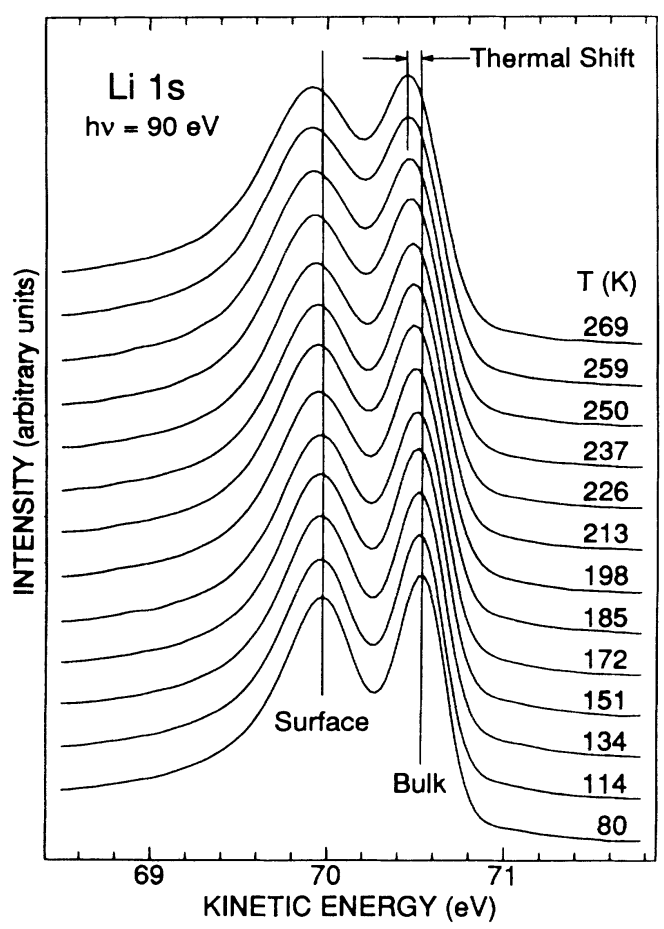

FIG. 3. Li $1 s$ photoemission spectra from the as-deposited (unannealed) Li surface, taken with $90-\mathrm{eV}$ photons as the sample warmed from 80 to $274 \mathrm{~K}$.

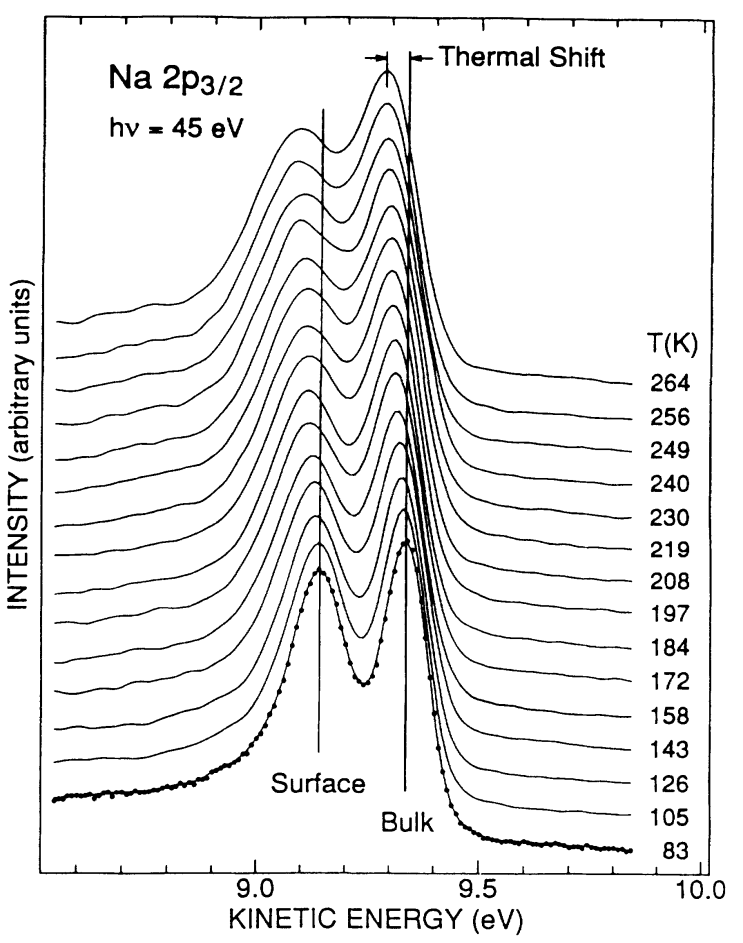

FIG. 4. Na $2 p_{3 / 2}$ photoemission spectra from the annealed $\mathrm{Na}$ surface, taken with $45-\mathrm{eV}$ photons. The Na $2 p_{1 / 2}$ contribution was removed by a subtractive stripping procedure.

latter extends into the region of the many-body tails of the bulk and surface lines and makes a significant contribution to the spectrum in the interval chosen for analysis.

The In data were fitted using two DS lines with com-

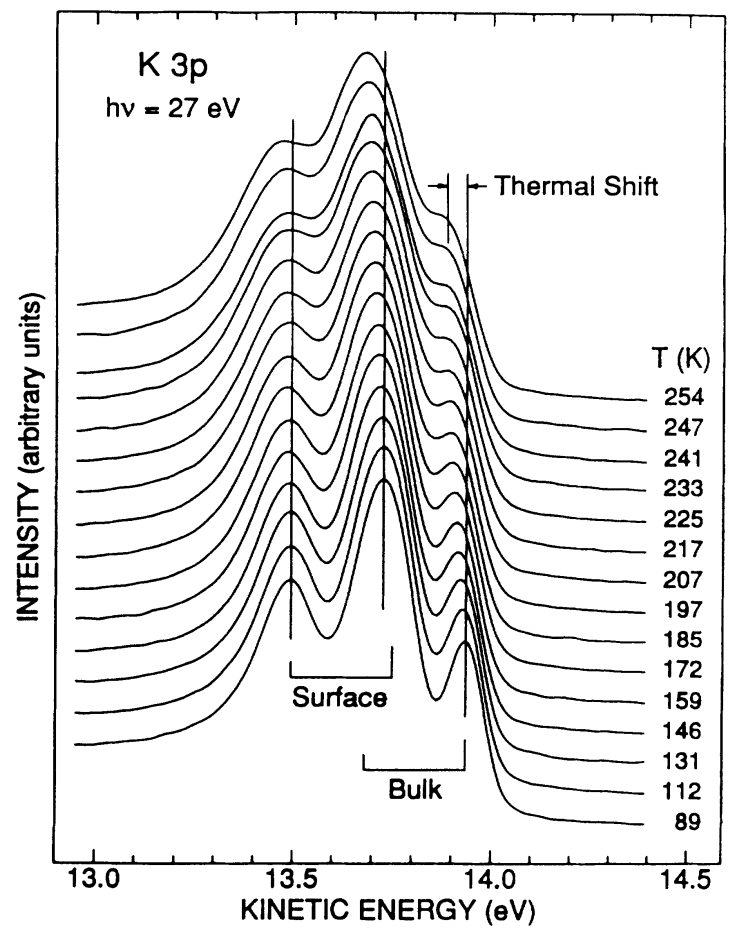

FIG. 5. K $3 p$ photoemission spectra from the as-deposited $\mathrm{K}$ surface, taken with $27-\mathrm{eV}$ photons. The spectra consist of overlapping bulk and surface doublets. 


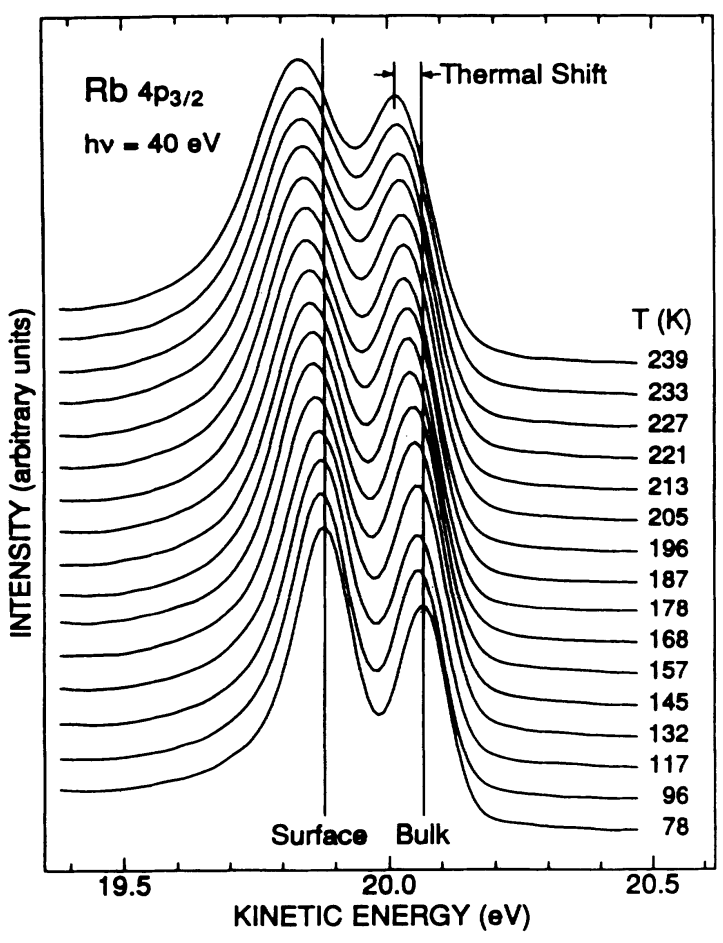

FIG. 6. $\mathrm{Rb} 4 p_{3 / 2}$ photoemission spectra from the asdeposited $\mathrm{Rb}$ surface, take with $40-\mathrm{eV}$ photons.

mon singularity index and lifetime width and a quadratic background. The spin-orbit splitting is $895 \pm 5 \mathrm{meV}$. The singularity index is $0.14 \pm 0.01$ and the lifetime width $0.14 \pm 0.01 \mathrm{eV}$. The Gaussian width of the $4 d_{5 / 2}$ line is significantly greater than that of the $4 d_{3 / 2}$ line, and both are much greater that the $100-\mathrm{meV}$ instrumental resolution. Although some of this width is due to phonon excitation, the excess broadening of this shallow $\frac{5}{2}$ level is very likely due to band dispersion.

\section{DISCUSSION}

\section{A. Thermal shifts}

The thermal shifts of the bulk lines obtained for the four alkali-metal samples as they warmed up from liquid-nitrogen to room temperature are shown in Fig. 7. The solid lines in the figure are the scaled thermal expansion of the metals, taken from the literature. ${ }^{10,11}$ (The thermal-expansion values on an absolute scale are shown in Fig. 8.) The good fit to the $\mathrm{Li}$ and $\mathrm{Na}$ data confirms that the shifts are indeed due to the expansion of the lattice. ${ }^{2,3}$ For $\mathrm{Rb}$, only the room-temperature value of the thermal-expansion coefficient is known, but since the Debye temperature of $\mathrm{Rb}(56 \mathrm{~K})$ is much smaller than that of $\mathrm{K}(100 \mathrm{~K})$, no significant curvature is expected in the temperature range of the data.

The thermal shift of the core-electron BE in a freeelectron metal $\Delta E_{\mathrm{BE}}$ arises from three contributions. ${ }^{12}$ The first is due to the change with thermal expansion of the Fermi level, which is the reference level for measured core-electron binding energies in a metal. The change in $E_{F}$ is due in part to the effect of thermal expansion on the Fermi energy, i.e., on the width of the occupied conduction band. In earlier work ${ }^{2}$ this quantity was approximated by a fraction $\left(\frac{3}{5}\right)$ of the thermal change in the Fermi energy. A more-exact result can be derived from the chemical potential, which includes the effects of exchange and correlation. We use an expression for the chemical potential relative to the average internal potential of a free-electron metal, ${ }^{13}$ a formalism applied in calculating work functions: ${ }^{14}$

$\bar{\mu}_{\mathrm{av}}=\frac{50.1}{r_{s}^{2}}-\frac{16.6}{r_{s}}-\frac{12.0}{r_{s}+7.8}\left(1+\frac{r_{s}}{3\left(r_{s}+7.8\right)}\right)$.

Here $r_{s}$ is the radius of a sphere containing one conduction electron, expressed in units of the Bohr radius $a_{0}$. The numerical constants in this and the following equations then give energies in units of eV. By differentiating Eq. (1) with respect to $r_{s}$, we obtain an expression for the change in Fermi level with thermal expansion,

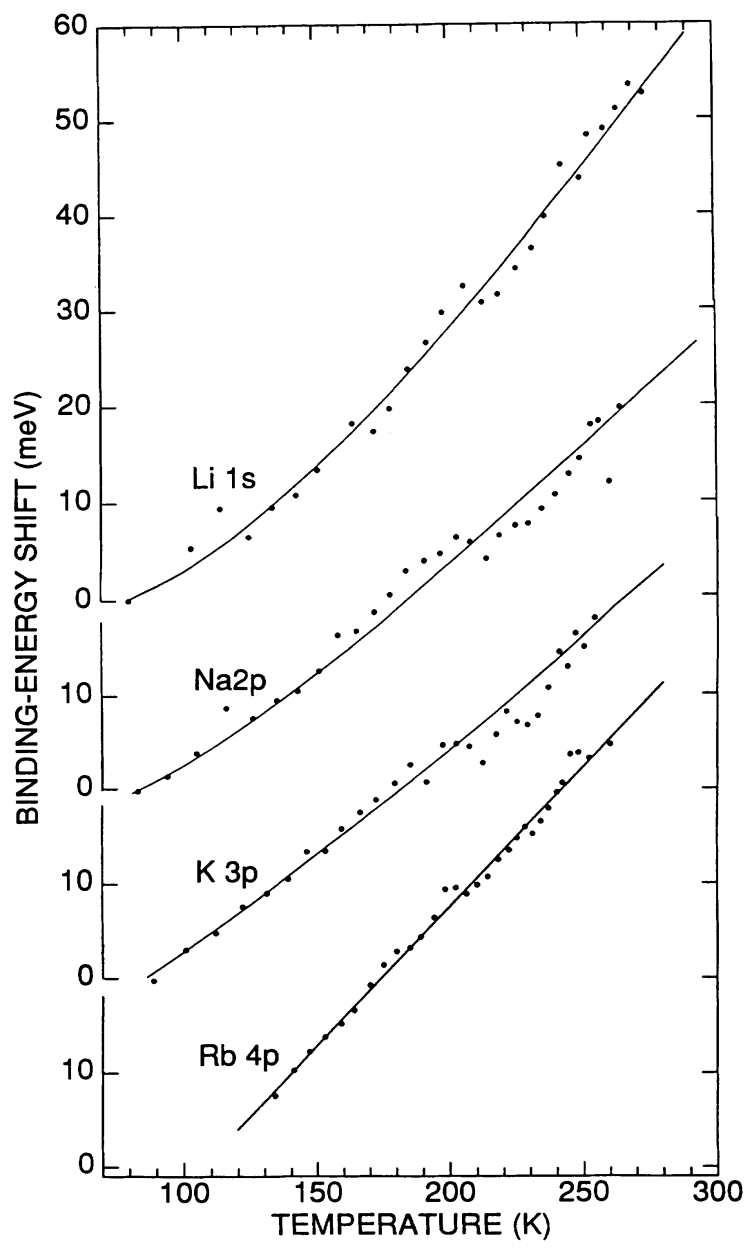

FIG. 7. Thermal shift of the bulk binding energies (dots), and scaled linear thermal expansion (solid lines) of $\mathrm{Li}, \mathrm{Na}, \mathrm{K}$, and $\mathrm{Rb}$. The fits were optimized by adjusting the vertical scale factor of the thermal expansion. 


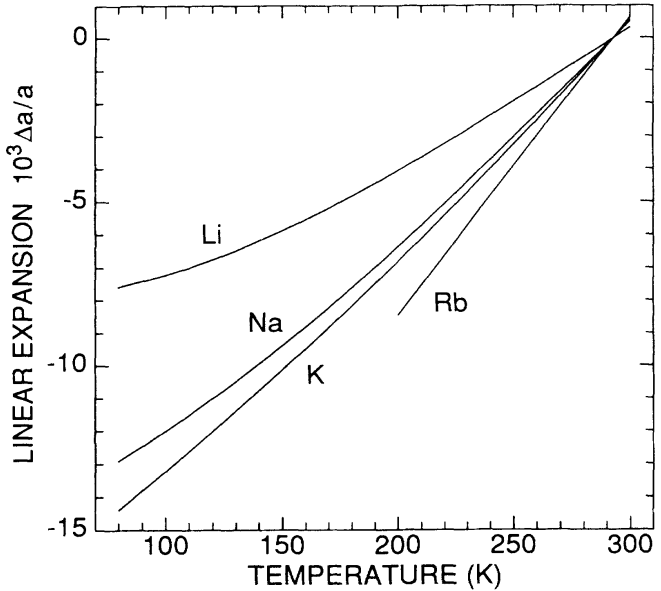

FIG. 8. Linear thermal expansion of four alkali metals relative to $293 \mathrm{~K}$. For $\mathbf{R b}$ only the room-temperature expansion coefficient is known.

$$
\begin{aligned}
\Delta E_{F}= & {\left[\frac{-100.2}{r_{s}^{2}}+\frac{16.6}{r_{s}}\right.} \\
& \left.+\frac{8.0 r_{s}}{\left(r_{s}+7.8\right)^{2}}\left[1+\frac{r_{s}}{r_{s}+7.8}\right]\right] \frac{\Delta a}{a},
\end{aligned}
$$

where we have replaced $\Delta r_{s} / r_{s}$ by $\Delta a / a$, the fractional thermal expansion. The negative term in the square brackets is equal to twice the Fermi energy.

The second contribution to the thermal shift arises from the reduction of the electrostatic potential within the atom due to the decrease in conduction-electron density with thermal expansion. In a monovalent metal with uniform conduction-electron density, the expression for this potential at radius $r$ in units of $a_{0}$ is ${ }^{15}$

$$
V(r)=\frac{e}{2 a_{0} r_{s}}\left[3-\frac{r^{2}}{r_{s}^{2}}\right], \quad r<r_{s} .
$$

Generalized for a polyvalent metal with $Z$ conduction electrons, this becomes

$$
V(r)=\frac{e}{2 a_{0} r_{s}}\left(3 Z^{2 / 3}-\frac{r^{2}}{r_{s}^{2}}\right) .
$$

The average potential inside the cell is

$$
V_{\mathrm{av}}=\frac{6 e Z^{2 / 3}}{5 a_{0} r_{s}},
$$

so that the potential relative to the average potential becomes

$$
V(r)-V_{\mathrm{av}}=\frac{e}{2 a_{0} r_{s}}\left(\frac{3}{5} Z^{2 / 3}-\frac{r^{2}}{r_{s}^{2}}\right) .
$$

Taking the derivative with respect to $r_{s}$, multiplying by $e$, and again replacing the fractional change in $r_{s}$ by $\Delta a / a$, we obtain the thermal shift due to the potential acting on the core electrons,

$$
\Delta E_{c}=\frac{8.16}{r_{s}}\left[Z^{2 / 3}-\frac{5 r^{2}}{r_{s}^{2}}\right) \frac{\Delta a}{a} .
$$

We have changed the sign in Eq. (7) because a decrease in the potential of the conduction electrons results in an increase in the core-electron BE.

The third and final contribution to the thermal shift is the temperature-dependent change of the relaxation energy. In an earlier paper, ${ }^{2}$ this effect was obtained from theoretical relaxation energies calculated by various methods. Here we determine this effect explicitly, starting with an expression for the relaxation energy (see the Appendix),

$$
E_{\mathrm{rel}}^{A}=\frac{e^{2} k_{0}}{2 \sqrt{1+k_{0} / k_{F}}},
$$

where $k_{0}$ and $k_{F}$ are the Thomas-Fermi and Fermi wave vectors, respectively. Expressed in terms of $r_{s}$, this becomes

$$
E_{\mathrm{rel}}^{A}=\frac{21.22}{r_{s}^{1 / 2}} \frac{1}{\left(1+0.815 r_{s}^{1 / 2}\right)^{1 / 2}}
$$

Differentiating with respect to $r_{s}$ gives the thermal change,

$$
\Delta E_{\mathrm{rel}}^{A}=\frac{10.61}{r_{s}^{1 / 2}} \frac{\left(1+1.222 r_{s}^{1 / 2}\right)}{\left(1+0.815 r_{s}^{1 / 2}\right)^{3 / 2}} \Delta a / a,
$$

where again the sign has been changed because decreased screening results in increased core-electron binding energy.

The sum of the three contributions to the thermal shift from Eqs. (2), (7), and (10) give the net calculated shift $\Delta E_{\mathrm{BE}}$. These values for the seven metals studied here are compared with the experimental values in Table I. The individual contributions and input parameters are also shown. The values of $r^{2}$ in Eq. (7) were set equal to $\left\langle r^{2}\right\rangle$ for the relevant core electron and were taken from Ref. 16. The agreement in magnitude between theory and experiment is good for all the metals except $\mathrm{Li}$ and $\mathrm{Al}$. Significantly, the theory does reproduce the correct sign in all cases. For Al the table shows that the negative shift has its origin in the large negative contribution of the Fermi level shift $\Delta E_{F}$. Referring to Eq. (2), it is seen to arise from the first term in square brackets, which is twice the Fermi energy, i.e., the negative thermal shift of $\mathrm{Al}$ is due to its large Fermi energy. The near cancellation of the $\mathrm{Mg}$ shifts also has its origin in this term. The poor agreement in the case of $\mathrm{Li}$ is of greater concern. A clue to the problem is found in recent experimental ${ }^{17}$ and theoretical $^{18}$ work, which found that the width of the occupied band is significantly smaller in $\mathrm{Na}$ and $\mathrm{Li}$ than the free-electron value. The discrepancy is largest for $\mathrm{Li}$, where the free-electron Fermi energy is $4.74 \mathrm{eV}$, while more sophisticated theory ${ }^{18}$ yields $2.75 \mathrm{eV}$. For $\mathrm{Na}$, the corresponding values are 3.24 versus $2.65 \mathrm{eV}$. If we simply insert the reduced values into the first term of Eq. (2), we obtain net thermal shifts of 51 and $68 \mathrm{meV}$ for $\mathrm{Li}$ and $\mathrm{Na}$, respectively, greatly improving the agreement with experiment for $\mathrm{Li}$ and bracketing the experimental value 
TABLE I. Thermal shifts in metals.

\begin{tabular}{|c|c|c|c|c|c|c|c|}
\hline & $\mathrm{Li}$ & $\mathrm{Na}$ & $\mathbf{K}$ & $\mathbf{R b}$ & $\mathrm{Mg}$ & $\mathrm{Al}^{\mathrm{a}}$ & In \\
\hline$r_{s}\left(a_{0}\right)$ & 3.25 & 3.93 & 4.86 & 5.20 & 2.66 & 2.07 & 2.41 \\
\hline $10^{3} \Delta a / a$ & 7.66 & 13.1 & 14.5 & $\sim 18$ & 4.68 & 12.32 & 6.05 \\
\hline$E_{\mathrm{rel}}(\mathrm{eV})^{\mathrm{b}}$ & 7.35 & 6.45 & 5.55 & & & 9.98 & \\
\hline$E_{\text {rel }}^{A}(\mathrm{eV})^{\mathrm{c}}$ & 7.49 & 6.62 & 5.76 & 5.50 & 8.53 & 10.01 & 9.08 \\
\hline$\Delta E_{F}(\mathrm{meV})$ & -31 & -26 & -7 & -3 & -36 & -187 & -61 \\
\hline$\Delta E_{c}(\mathrm{meV})$ & 15 & 21 & 13 & 12 & 17 & 77 & 16 \\
\hline$\Delta E_{\mathrm{rel}}^{A}(\mathrm{meV})$ & 37 & 57 & 55 & 66 & 26 & 78 & 35 \\
\hline$\Delta E_{\mathrm{BE}}(\mathrm{meV})$ (calc.) & 21 & 52 & 61 & 75 & 7 & -32 & -10 \\
\hline$\Delta E_{\mathrm{BE}}(\mathrm{meV})$ (expt.) & 60 & 58 & 47 & 63 & $\sim 0^{\mathrm{d}}$ & -76 & -3 \\
\hline
\end{tabular}

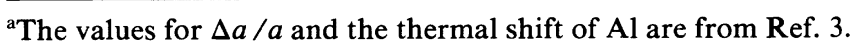

${ }^{b}$ Without correction for the finite extent of the core-electron wave function, from Ref. 4.

${ }^{\mathrm{c}}$ From Eq. (9).

${ }^{\mathrm{d}}$ Reference 2.

for Na. While this procedure cannot be defended in detail because other $r_{s}$-dependent terms would presumably also need to be modified, it does indicate that the failure of the free-electron model in the calculation of the thermal shift for $\mathrm{Li}$ is related to its deviations from freeelectron character, and does not impugn the validity of Eqs. (2), (7), and (10) for the contributions to the thermal shift.

The data in Figs. 3-6 show that the thermal shift of the surface atoms is quite comparable to that of the bulk atoms. As discussed below, the free-electron model for the thermal shift explains this result in terms of the relatively small band narrowing at the surface of the alkali metals.

\section{B. Alkali-metal surface-atom core-level shifts}

The present data provide the most accurate determination of the surface-atom core-level shift at the alkalimetal (110) surfaces. Values of the SCS's obtained from our analysis are $0.54,0.19,0.20$, and $0.19 \mathrm{eV}$ for $\mathrm{Li}, \mathrm{Na}$, $\mathrm{K}$, and $\mathrm{Rb}$, respectively. Cs has a SCS of $0.23 \mathrm{eV},{ }^{19}$ slightly larger than those reported here for $\mathrm{Na}, \mathrm{K}$, or $\mathrm{Rb}$.

Earlier measurements of the alkali-metal SCS's are, with the exception of $\mathrm{Li}$, in only fair agreement with the more-reliable values obtained here. Recent determinations of the SCS of $\mathrm{Li}$ (Ref. 20) gave a shift of $0.55 \mathrm{eV}$, in very good agreement with our result. The shift of 0.22 $\mathrm{eV}$ reported for $\mathrm{Na},{ }^{21}$ however, is not consistent with the data in Fig. 4 or with the smaller value reported in Ref. 7. The erroneously large value for the shift is correlated with the erroneously small spin-orbit splitting obtained in Ref. 21. For K, shifts of 0.23 (Ref. 22) and $0.25 \mathrm{eV}$ (Ref. 23) have been reported. These shifts are not compatible with the data in Fig. 5, demonstrating the difficulty of extracting parameters from overlapping spin-orbit doublets. For Rb, shifts of 0.24 (Ref. 22) and $0.19 \mathrm{eV}$ (Ref. 24) have been reported. The former is significantly larger than the value reported here, while the latter, based on photoemission with $\mathrm{He}$ II resonance radiation, is in agreement with the present results. These comparisons clearly under- score the need for high resolution in determining corelevel BE shifts at surfaces.

An expression for the surface-atom core-level shift $\Delta_{s, b}$ of the $Z$ metal in terms of bulk and surface cohesive and implantation energies of the $Z$ and $Z+1$ metals has been derived from a Born-Haber thermodynamic cycle: ${ }^{25}$

$$
\begin{aligned}
\Delta_{s, b}= & \left(E_{\text {coh,bulk }}^{Z+1}-E_{\text {coh,surf }}^{Z+1}\right) \\
& -\left(E_{\text {coh, bulk }}^{Z}-E_{\text {coh, surf }}^{Z}\right) \\
& -\left(E_{\text {impl,bulk }}^{Z+1 \rightarrow Z}-E_{\text {impl, surf }}^{Z+1 \rightarrow Z}\right) .
\end{aligned}
$$

A popular approximation for close-packed surfaces is that the surface cohesive and implantation energies are $80 \%$ of the bulk values, ${ }^{25}$ which simplifies Eq. (11) to

$$
\Delta_{s, b}=0.2\left(E_{\text {coh, bulk }}^{Z+1}-E_{\text {coh,bulk }}^{Z}-E_{\text {impl,bulk }}^{Z+1 \rightarrow Z}\right) \text {. }
$$

This assumption is based on the fact that the nearestneighbor coordination numbers of atoms in the closepacked surfaces of bcc, fcc, and hcp lattices are $75 \%$ of the bulk coordination numbers. Note that $E_{\mathrm{impl}}$ is the energy required to do the implantation, and so may be approximated by the molar heat of solution of the $Z+1$ element in the $Z$ metal. Using tabulated values for $E_{\text {coh }}$ (Ref. 26) and semiempirical values for $E_{\text {impl }}$ [Eq. (6) in Ref. 27] (see Table II) yields SCS's for Li, Na, K, Rb, and Cs of $0.18,0.00,0.10,0.10$ and $0.13 \mathrm{eV}$, respectively. While these thermodynamic estimates do produce the correct sign and relative sizes of the surface-atom shifts, the agreement with the experimental results is far from gratifying. The disagreement is, however, no worse than that found for the (110) surfaces of the bcc transition metals $\mathrm{W}$ and Ta. ${ }^{25,28}$ These comparisons highlight the inadequacy of the approximation made in deriving Eq. (12). The fault lies not in the Born-Haber cycle in Eq. (11), but in the assumption regarding the surface cohesive energy.

The use of the $80 \%$ reduction factor to approximate $E_{\text {coh,surf }}$ energy can be avoided if the surface energies of the $Z$ and $Z+1$ metals are known. Equation (11) is then 
TABLE II. Parameters for calculation of surface shifts (eV/atom) and surface-atom core-level shifts (eV).

\begin{tabular}{|c|c|c|c|c|c|c|c|c|c|}
\hline \multicolumn{2}{|c|}{ Element } & \multicolumn{2}{|c|}{$\begin{array}{l}\text { Cohesive } \\
\text { energy }^{\mathrm{a}}\end{array}$} & \multicolumn{2}{|c|}{$\begin{array}{l}\text { Surface } \\
\text { energy }^{b}\end{array}$} & \multirow{2}{*}{$\begin{array}{c}\begin{array}{c}\text { Implantation } \\
\text { energy }^{c}\end{array} \\
Z+1 \rightarrow Z\end{array}$} & \multicolumn{3}{|c|}{$\begin{array}{l}\text { Surface-atom } \\
\text { core-level shift }\end{array}$} \\
\hline$Z$ & $Z+1$ & $Z$ & $Z+1$ & $Z$ & $Z+1$ & & Expt. & Eq. (12) & Eq. (13) \\
\hline $\mathrm{Li}$ & $\mathrm{Be}$ & 1.64 & 3.32 & 0.28 & 0.46 & 0.80 & 0.54 & 0.18 & 0.08 \\
\hline $\mathrm{Na}$ & $\mathrm{Mg}$ & 1.11 & 1.50 & 0.21 & 0.44 & 0.40 & 0.19 & 0.00 & 0.18 \\
\hline $\mathrm{K}$ & $\mathrm{Ca}$ & 0.93 & 1.84 & 0.18 & 0.42 & 0.41 & 0.20 & 0.10 & 0.20 \\
\hline $\mathrm{Rb}$ & $\mathrm{Sr}$ & 0.85 & 1.72 & 0.16 & 0.42 & 0.36 & 0.19 & 0.10 & 0.21 \\
\hline Cs & $\mathrm{Ba}$ & 0.80 & 1.90 & 0.15 & 0.42 & 0.42 & 0.23 & 0.13 & 0.22 \\
\hline $\mathrm{Mg}$ & $\mathrm{Al}$ & 1.50 & 3.39 & 0.44 & 0.51 & -0.05 & 0.14 & 0.39 & 0.07 \\
\hline $\mathrm{Al}$ & $\mathrm{Si}$ & 3.39 & 4.62 & 0.51 & 0.45 & -0.08 & -0.06 & 0.26 & -0.04 \\
\hline In & Sn & 2.52 & 3.12 & 0.58 & 0.70 & -0.01 & $<0.1$ & 0.12 & 0.12 \\
\hline
\end{tabular}

${ }^{\mathrm{a}}$ Reference 26.

${ }^{\mathrm{b}}$ Reference 29.

${ }^{\mathrm{c}}$ Reference 27.

expressed directly in terms of surface energies ( $E_{\text {surf }}=E_{\text {coh, bulk }}-E_{\text {coh, surf }}$ ), yielding

$$
\Delta_{s, b}=E_{\text {surf }}^{Z+1}-E_{\text {surf }}^{Z}-\left(E_{\text {impl,bulk }}^{Z+1 \rightarrow Z}-E_{\text {impl, surf }}^{Z+Z}\right) .
$$

Low-temperature solid-phase surface energies, derived from liquid-phase surface-energy measurements, have been determined for a wide variety of elemental materials, ${ }^{29}$ leaving only the surface implantation energy undetermined in Eq. (13). In evaluating this equation we have approximated the difference between the bulk and surface implantation energy by $12 \%$ of the molar heat of solution of the $Z+1$ element in the $Z$ metal. This factor was chosen to optimize the agreement with the experimental data. Using this approach, we obtain shifts of $0.08,0.18,0.20,0.21$, and $0.22 \mathrm{eV}$ for $\mathrm{Li}, \mathrm{Na}, \mathrm{K}, \mathrm{Rb}$, and $\mathrm{Cs}$, respectively. Except for $\mathrm{Li}$, these results are in very good agreement with the experimental results. The error in the case of $\mathrm{Li}$ is probably due to the small surface energy obtained for the close-packed surface of the hexagonal $Z+1$ metal Be. Note that its surface energy is only $14 \%$ of the bulk cohesive energy, while those of the other $Z+1$ metals average $25 \%$. Scaling up the Be surface energy accordingly yields a surface-atom core-level shift of $0.44 \mathrm{eV}$, in much better agreement with experiment. An examination of the input parameters summarized in Table II shows that most of the error in the use of Eq. (12) arises from an underestimate of the surface energy of the alkaline-earth metals combined with a slight overestimate of the surface energy of the alkali metals.

Table II also includes input parameters and shifts calculated from Eqs. (12) and (13) for $\mathrm{Mg}, \mathrm{Al}$, and In. For $\mathrm{Mg}$, a shift of $0.14 \mathrm{eV}$ has been reported, ${ }^{21}$ based on the analysis of broadened spectra in which the surface component is not resolved. This shift is fairly close to the value of $0.07 \mathrm{eV}$ obtained from Eq. (13), but is much smaller than the value of $0.39 \mathrm{eV}$ obtained from Eq. (12). The predicted shift of $-0.04 \mathrm{eV}$ for $\mathrm{Al}$ is in excellent agreement with a reported value of $-0.06 \mathrm{eV}$ for Al(100). ${ }^{30}$ The shift obtained from Eq. (12) is very much larger and of opposite sign. For In, the data in Fig. 2 indicate only that the shift is less than $0.1 \mathrm{eV}$. In this case,
Eqs. (12) and (13) agree in yielding a shift of $0.12 \mathrm{eV}$. Overall, it is clear that Eq. (13) is much more successful in predicting SCS's than Eq. (12).

While this agreement between experiment and the Born-Haber cycle is gratifying, it provides no insight into the microscopic, electronic origin of the SCS in freeelectron-like metals. Recall that the surface core-level shifts for noble and transition metals ${ }^{28}$ could be understood in terms of the narrowing of the tight-binding $d$ band about its centroid due to the reduced coordination at the surface. This results in a small charge flow from the surface into the bulk conduction band, or vice versa, depending on whether the band is less or more than half filled. As a result, metals near the left side of the transition-metal series exhibit positive SCS's, while those near the right are negative. Although this model does yield the correct sign for the SCS of the alkali metals, it is not applicable to them because they have free-electronlike conduction bands. Moreover, our observations here of thermal shifts for surface atoms that are comparable to those of the bulk atoms indicates through Eq. (2) that the bandwidth of the surface atoms is not very different from that of the bulk. Independent evidence that the surface conduction band of the alkali metals is not significantly narrower than that for the bulk is obtained by comparing the surface-sensitive, angle-resolved photoemission data from $\mathrm{Na}$ (Ref. 17) with the bulk-sensitive absorption-edge data: ${ }^{31}$ the two measurements yield comparable bulk band narrowing (due to correlation effects), with no indication of additional narrowing at the surface.

We now show that our analysis above of the thermal shifts provides the insight necessary to develop a description of the microscopic, electronic origin of the surfaceatom core-level shift. In general, the reduced coordination of an atom at the surface of a metal results not only in some band narrowing, but also in a spill-out of valence-band charge into the vacuum. (The latter produces the dipole layer, which is responsible for part of the work function.) Both of these effects are similar to those produced by thermal expansion in a free-electron-like metal. The band narrowing lowers the Fermi level and decreases the core-level binding energy of the surface 
atoms. Acting in the opposite direction is the change in the potential due to the spill-out of the valence electrons, which increases the binding energy. In the case of $\mathrm{Na}$, for example, we have cited evidence that the band narrowing at the surface is very small, so that the effect of the decreased potential dominates, resulting in a large positive SCS. For the divalent and trivalent metals with larger Fermi energies, band narrowing becomes more important, resulting in smaller positive or even negative SCS's. Table II bears this out, showing that the alkali metals with comparatively small Fermi energies do indeed have the most positive shifts. Within this description, then, the potential term dominates in the alkali metals, while in the larger-bandwidth $s p$ metals this term is largely negated by the Fermi-energy term, much the same as is the case of the thermal induced changes. It is, of course, likely that there is also a contribution from a change in relaxation energy at the surface, but it is more difficult to estimate.

It is worth emphasizing that the above description of the surface-atom core-level shift in the alkali metals differs in a number of aspects from that given previously for the noble and transition metals. ${ }^{28}$ For the latter, it was argued that the shift originates from the narrowing of the tight-binding $d$ band about its centroid, which was assumed fixed relative to the core levels. A small charge flow between the surface layer and the bulk is sufficient to bring the surface Fermi level into coincidence with that of the bulk. The contribution from the change in potential due to the spill-out of the conduction electrons at the surface was ignored. For the alkali metals, the narrowing of the conduction band makes a relatively small contribution to the SCS compared with the effects of the spill-out of the conduction electrons.

The present results imply that the potential shift should also be considered in the $d$-band metals, where it would contribute a positive term to the SCS. It would be interesting to extend measurements of surface and bulk thermal shifts to the tight-binding metals to test further these simple concepts.

\section{CONCLUSIONS}

We have confirmed that the thermal shift of coreelectron BE's is proportional to the lattice expansion. It contains two initial-state contributions: a negative one from the effect of thermal expansion on the Fermi energy, and a positive one from the reduction of the conductionelectron charge density. In addition, there is a positive final-state contribution from the decrease in relaxation energy. In monovalent alkali metals the positive terms dominate, in divalent alkaline-earth metals the terms tend to cancel, and in the trivalent metals the larger Fermi energy makes the shift negative. We have also obtained more-reliable values for the SCS's of the alkali metals and have demonstrated that they are compatible with known surface energies. The SCS's in free-electronlike metals can be understood within the same electronic framework as the thermally induced shifts.

\section{ACKNOWLEDGMENTS}

We are indebted to Y. Yafet, L. F. Mattheiss, and M. Schlüter for stimulating discussions, and to D. A. Papaconstantopoulos for numerical results of bandstructure calculations for the alkali metals. The alkalimetal photoemission research was carried out at the $\mathrm{Na}$ tional Synchrotron Light Source (NSLS), Brookhaven National Laboratory, which is supported by the Department of Energy, Division of Materials Sciences and Division of Chemical Sciences.

\section{APPENDIX}

We derive an approximate expression for the relaxation (or extra-atomic polarization) energy of the core levels in a simple metal. As obtained by Hedin and Lundqvist in the Hartree-Fock formalism ${ }^{4}$ and by Lang and Williams in the density-functional formalism, ${ }^{32}$ the increase in relaxation energy beyond the intra-atomic contribution (which is assumed unchanged in going from the free-atom to the metallic environment) due to screening by the free carriers of the metal can be simply expressed as

$$
E_{\mathrm{rel}}=\frac{1}{2} e \phi_{e a}(r=0) .
$$

Here $\phi_{e a}$ is the potential from the extra-atomic screening charge at the position $r=0$ of the core level. This expression obviously ignores the spatial extent of the core levels and thus slightly overestimates the magnitude of the relaxation energy. In general, within the linear-response regime, $\phi_{e a}(0)$ can be written as

$$
\begin{aligned}
\phi_{e a}(0)=\int_{0}^{\infty} \frac{q^{2}}{2 \pi^{2}} & \left(\frac{1}{1-\left(4 \pi / q^{2}\right) \hat{\chi}(q)}-1\right) \\
& \times \hat{\phi}_{\text {core }}(q) d q,
\end{aligned}
$$

where $\hat{\phi}(q)$ and $\hat{\chi}(q)$ are the momentum-space representations of the core potential and susceptibility of the metal, respectively. Since we are ignoring the spatial distribution of the core level, $\hat{\phi}(q)$ is simply $4 \pi e / q^{2}$. The simplest approximation for $\chi(q)$ is the Thomas-Fermi expression $\chi_{\mathrm{TF}}(q)=-k_{0} / 4 \pi$, where $k_{0} a_{0}=1.56 / r_{s}^{1 / 2}$. Using this, the relaxation energy can be expressed as

$$
E_{\mathrm{rel}}^{\mathrm{TF}}=\frac{e^{2}}{2 a_{0}} k_{0} a_{0}=\frac{21.22}{r_{s}^{1 / 2}} .
$$

Compared to values obtained by Hedin and Lundqvist, ${ }^{4}$ Eq. (A3) tends to overestimate $E_{\text {rel }}$ by $40-70 \%$ for metallic densities. This is because the Thomas-Fermi susceptibility significantly overestimates the ability of the Fermi sea to screen out short-wavelength perturbations. A more-accurate susceptibility has been derived in the random-phase approximation (RPA), which describes the reduced response at higher momenta. It can be written as $^{33}$

$$
\chi_{\mathrm{RPA}}(q)=\chi_{\mathrm{TF}}(q)\left(\frac{1}{2}+\frac{1-x^{2}}{4 x} \ln \left|\frac{1+x}{1-x}\right|\right),
$$

where $x=q / 2 k_{F}$ and $k_{F}=1.227 k_{0} / r_{s}^{1 / 2}$ is the Fermi momentum. This susceptibility, however, does not lead 


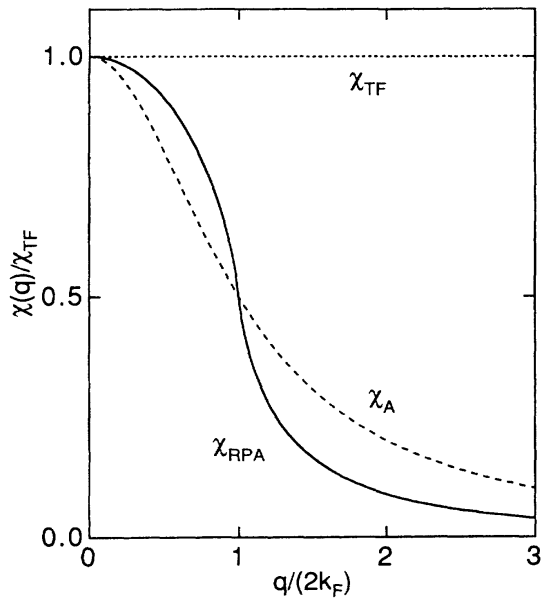

FIG. 9. Approximations to the momentum-space representation of the susceptibility of a free-electron metal in units of $-k_{0} / 4 \pi$, which is the Thomas-Fermi susceptibility, $\chi_{\mathrm{TF}}$. Also shown are the result of the random-phase approximation $\chi_{\mathrm{RPA}}$ and the approximation used here, $\chi_{A}$.

to an analytic form for the relaxation energy versus $r_{s}$.

In order to overcome the difficulties produced by both the TF and RPA susceptibilities in driving useful and accurate expressions for $E_{\text {rel }}$, we introduce the following approximate form for the susceptibility:

$$
\chi_{A}(q)=\chi_{\mathrm{TF}}(q) \frac{1}{1+x^{2}} .
$$

Although it is simple in form, it has the desired properties of reducing to the Thomas-Fermi form for small $q$ and approaching 0 for large $q$, as does the RPA susceptibility. In addition, it matches the value of $\chi_{\mathrm{RPA}}$ at the critical value of $q=2 k_{F}$, where the response has dropped to $\frac{1}{2}$ of its long-wavelength value. As shown in Fig. 9, which plots all three versions of the susceptibility, $\chi_{A}(q)$ does a reasonable job of mimicking $\chi_{\mathrm{RPA}}(q)$. Using $\chi_{A}(q)$, we obtain

$$
E_{\mathrm{rel}}^{A}=E_{\mathrm{rel}}^{\mathrm{TF}} \frac{1}{\left(1+k_{0} / k_{F}\right)^{1 / 2}}
$$

In row 4 of Table I, $E_{\text {rel }}^{A}$ from Eq. (A6) is displayed for the metals under consideration. Also shown are values calculated in Ref. 4 for $\mathrm{Al}, \mathrm{Li}, \mathrm{Na}$, and $\mathrm{K}$, also ignoring the spatial extent of the core levels. For these four metals, $E_{\text {rel }}^{A}$ reproduces the values of Ref. 4 to better than $4 \%$. If the spatial extent of the shallow core levels of these metals is taken into account, then the screening energies are reduced from a minimum of $4 \%$ for $\mathrm{Al}$ up to a maximum of $23 \%$ for $\mathrm{K}^{4}$ However, because this reduction tends to be offset by the nonlinear response of the charge density to the potential, ${ }^{34}$ which has not been considered here, we use Eq. (10) [derived from Eq. (A6)] as it stands in the evaluation of the change in relaxation energy with thermal expansion.

${ }^{*}$ Present address: Department of Physics, University of Texas, Austin, TX 78712.

${ }^{1}$ M. A. Butler, G. K. Wertheim, D. L. Rousseau, and S. Hüfner, Chem. Phys. Lett. 13, 473 (1972).

${ }^{2}$ P. H. Citrin, G. K. Wertheim, and Y. Baer, Phys. Rev. B 16, 4256 (1977).

${ }^{3}$ J. A. Tagle, E. T. Arakawa, and T. A. Callcott, Phys. Rev. B 21, 4552 (1980).

${ }^{4}$ L. Hedin and S. Lundqvist, in Solid State Physics, edited by F. Seitz, D. Turnbull, and H. Ehrenreich (Academic, New York, 1969), Vol. 23, p. 1.

${ }^{5}$ D. M. Riffe, G. K. Wertheim, and P. H. Citrin, Phys. Rev. Lett. 63, 1976 (1989).

${ }^{6}$ B. S. Itchkawitz, I.-W. Lyo, and E. W. Plummer, Phys. Rev. B 41, 8075 (1990).

${ }^{7}$ D. M. Riffe, G. K. Wertheim, and P. H. Citrin, Phys. Rev. Lett. 67, 116 (1991).

${ }^{8}$ S. Doniach and M. Šunjić, J. Phys. C 3, 285 (1970).

${ }^{9}$ M. Kato, Phys. Rev. B 38, 10915 (1988).

${ }^{10}$ American Institute of Physics Handbook, edited by D. E. Gray (McGraw-Hill, New York, 1972), pp. 4-126.

${ }^{11}$ D. R. Schouten and C. A. Swenson, Phys. Rev. B 10, 2175 (1974).

${ }^{12}$ There is an additional shift of instrumental origin whenever the temperature of the sample is not equal to that of the spectrometer. It is due to the thermal emf of the circuit element sustaining the temperature gradient, usually a copper grounding wire. It will produce a shift of only $0.3 \mathrm{meV}$ between 78 and $295 \mathrm{~K}$.

${ }^{13}$ J. Hölzl and F. K. Schulte, in Solid Surface Physics, edited by G. Höler, Springer Tracts in Modern Physics Vol. 85 (Springer, Berlin, 1979), p. 1.

${ }^{14}$ N. D. Lang and W. Kohn, Phys. Rev. B 3, 1215 (1971); 8, 6010 (1973).

${ }^{15}$ J. Bardeen, Phys. Rev. 52, 688 (1937).

${ }^{16}$ C. Lu, T. A. Carlson, F. B. Malik, T. C. Tucker, and C. W. Nestor, Jr., At. Data 3, 1 (1971).

${ }^{17}$ I.-W. Lyo and E. W. Plummer, Phys. Rev. Lett. 60, 1558 (1988); see also E. Jensen and E. W. Plummer, Phys. Rev. Lett. 55, 1912 (1985).

${ }^{18}$ J. E. Northrup, M. S. Hybertsen, and S. G. Louie, Phys. Rev. Lett. 59, 819 (1987).

${ }^{19}$ G. K. Wertheim and D. N. E. Buchanan, Phys. Rev. B 43, 13815 (1991).

${ }^{20}$ M.-L. Shek, J. Hrbeck, T. K. Sham, and G.-Q. Xu, Phys. Rev. B 41, 3447 (1990).

${ }^{21}$ R. Kammerer, J. Barth, F. Gerken, C. Kunz, S. A. Flodstrom, and L. I. Johansson, Phys. Rev. B 26, 3491 (1982).

${ }^{22}$ T. K. Sham and J. Hrbeck, J. Chem. Phys. 89, 1188 (1988); T. K. Sham, G.-Q. Xu, J. Hrbeck, and M. L. Shek, Surf. Sci. 210, L185 (1989).

${ }^{23}$ R. L. Fink, P. N. First, and C. P. Flynn, Phys. Rev. B 38, 5839 (1988).

${ }^{24}$ G. K. Wertheim, D. N. E. Buchanan, and J. E. Rowe, Solid State Commun. 77, 903 (1991).

${ }^{25}$ B. Johansson and N. Mårtensson, Phys. Rev. B 21, 4427 (1980).

${ }^{26}$ C. Kittel, Introduction to Solid State Physics, 5th ed. (Wiley, New York, 1976), p. 74.

${ }^{27}$ A. R. Miedema, P. F. de Châtel, and F. R. de Boer, Physica 100B, 1 (1980).

${ }^{28}$ P. H. Citrin and G. K. Wertheim, Phys. Rev. B 27, 3176 (1983), and references cited therein.

${ }^{29}$ We use values from W. R. Tyson and W. A. Miller, Surf. Sci. 
62, 267 (1977). See also A. R. Miedema, Z. Metallkd. 69, 287 (1978). Surface energies in Table II have been converted from $\mathrm{erg} / \mathrm{cm}^{2}$ to $\mathrm{eV} /$ atom for the close-packed surfaces of the metals listed. Although it is not obvious that solid-phase energies deduced from liquid-phase data should correspond to closepacked-surface (CPS) surface energies, the good agreement of the derived values with recent density-functional CPS surface-energy calculations of simple metals [D. Zhang, D. C. Langreth, and J. P. Perdew, Phys. Rev. B 41, 5674 (1990)] suggests that the errors are insignificant.

${ }^{30}$ We quote the partial-yield SCS value of T.-C. Chiang, and D. E. Eastman, Phys. Rev. B 23, 6836 (1981), taken with the highest resolution to date $(70 \mathrm{meV})$. The (111) surface, not the (100) surface, is the close-packed surface for Al; therefore, the estimation from Eq. (13) is perhaps better than indicated, since the $A l(111)$ surface does indeed have a smaller, albeit undetermined, shift than that of the $\mathrm{Al}(100)$ surface (see Ref. 21).

${ }^{31}$ P H. Citrin, G. K. Wertheim, T. Hashizume, F. Sette, A. A. MacDowell, and F. Comin, Phys. Rev. Lett. 61, 1021 (1988).

${ }^{32}$ N. D. Lang and A. R. Williams, Phys. Rev. B 16, 2408 (1977).

${ }^{33}$ See, e.g., N. W. Ashcroft and N. D. Mermin, Solid State Physics (Holt, Rinehart and Winston, New York, 1976), p. 343.

${ }^{34}$ C.-O. Almbladh and U. Barth, Phys. Rev. B 13, 3307 (1976). 